\title{
Triple kinase inhibitor with phosphodiesterase-5 inhibitor for idiopathic pulmonary fibrosis
}

\author{
Tomoo Kishaba \\ Department of Respiratory Medicine, Okinawa Chubu Hospital, Uruma City, Okinawa, Japan \\ Correspondence to: Tomoo Kishaba. Department of Respiratory Medicine, Okinawa Chubu Hospital, 281 Miyazato, Uruma City, Okinawa 904-2293, \\ Japan. Email: kishabatomoo@gmail.com. \\ Comment on: Kolb M, Raghu G, Wells AU, et al. Nintedanib plus Sildenafil in Patients with Idiopathic Pulmonary Fibrosis. N Engl J Med 2018. [Epub \\ ahead of print].
}

Submitted Oct 17, 2018. Accepted for publication Oct 22, 2018.

doi: $10.21037 /$ jtd.2018.10.84

View this article at: http://dx.doi.org/10.21037/jtd.2018.10.84

Idiopathic pulmonary fibrosis (IPF) is relentless progressive parenchymal disease (1), and median survival is 2-3 years (2). Natural course of IPF patients is variable from stable disease, partly reversible, progressive deterioration to acute exacerbation (AE). IPF patients show significant decline of lung function especially both forced vital capacity (FVC) and diffusion capacity of the lungs for carbon monoxide (DLco) (3). Prediction of pulmonary function test (PFT) decline can be possible for early mortality of IPF (4). However, we cannot predict long-term decline of PFT for IPF patients easily. FVC trend is often associated with dyspnea grade (5). Total lung capacity, transfer factor, arterial partial pressure of oxygen at rest, the lowest oxygen saturation during exercise test, and baseline dyspnea index (BDI) score were significantly correlated with the total St. George's Respiratory Questionnaire (SGRQ) score. Dyspnea was the most important factor determining health-related quality of life (HRQoL) in IPF patients (6). Therefore, both reduction of decline of FVC and mitigation of dyspnea grade provide comfortable life for IPF patients. Traditionally, prednisolone and immunosuppressants such as cyclophosphamide and cyclosporine have been used for chronic management of IPF $(7,8)$. These anti-inflammatory agents have been used based on the theory of the main pathogenesis is inflammation in IPF patients (1). However, it has been clarified that recurrent alveolar epithelial injury and sequential aberrant repair mechanism contribute to myoblast recruitment and deposition of extracellular matrix (9). According to recent theory of the main mechanism and the positive results of international clinical trials for IPF, two anti-fibrotic agents such as pirfenidone (PFD) and nintedanib have been approved for IPF patients (10-12). ASCEND study consisted of 1,247 mild to moderate IPF patients. At 1 year, PFD reduced the proportion of patients with significant decline of percent predicted $\mathrm{FVC}(\% \mathrm{FVC}$ ) by $43.8 \%$ and increased the proportion of patients with no decline of $\% \mathrm{FVC}$ by $59.3 \%$. A treatment benefit was also observed for progression-free survival, 6-min walk distance and dyspnea grade. Regarding long-term effect of PFD for IPF patients, Bando et al. showed the patients who took PFD treatment for 2 years or longer were 111 cases $(22.1 \%)$ out of 502 patients. The mean change in the FVC was $-30 \pm 224 \mathrm{~mL}$ in the first year of treatment, $-158 \pm 258 \mathrm{~mL}$ in the second year, and $-201 \pm 367 \mathrm{~mL}$ in the third year. FVC improved by $10 \%$ or more in the first year in 10 (14.7\%) of 68 cases, and showed stability in 47 (69.1\%) cases. FVC stable cases in the second and third years were $61.7 \%$ and $62.5 \%$ of the patients, respectively (13). Another study, Ogawa et al. evaluated that $46 \mathrm{IPF}$ patients who received PFD over 1 year. The median age and baseline \% FVC of this study were 70.5 years and $70.0 \%$, respectively. FVC changes in patients who took long-term were -120 and $-170 \mathrm{~mL}$ at 12 and 24 months after receiving PFD, respectively. Median survival times of the patients who took PFD therapy in the long-term was 1,612 days, which was significantly longer compared to short term 285 days $(\mathrm{P}<0.001)$. AE free survival of longterm group was longer than those in short-term group in this study (947 vs. 145 days, $\mathrm{P}=0.001)$ (14). Nintedanib is a triple kinase inhibitor which inhibit fibroblast growth factor, 
platelet-derived growth factor, and vascular endothelial growth factor receptors (15). Nintedanib was initially developed anti-cancer drugs especially for adenocarcinoma. There have been accumulated robust evidence of antifibrotic action in vitro and in vivo. Based on the positive results of a phase II study (12), a phase III study was conducted, in which 1,066 IPF patients were randomly assigned in a 3:2 ratio to receive nintedanib or placebo. The adjusted annual change of FVC was $-114.7 \mathrm{~mL}$ with nintedanib versus $-239.9 \mathrm{~mL}$ with placebo (difference, $125.3 \mathrm{~mL} ; \mathrm{P}<0.001)$ in INPULSIS- 1 and $-113.6 \mathrm{~mL}$ with nintedanib versus $-207.3 \mathrm{~mL}$ with placebo (difference, $93.7 \mathrm{~mL}$; $<<0.001$ ) in INPULSIS-2. In INPULSIS-1, there was no significant difference between the nintedanib and placebo groups in $\mathrm{AE}$ free survival time [hazard ratio (HR) with nintedanib, $1.15 ; \mathrm{P}=0.67$ ]; in INPULSIS-2, there was a significant benefit with nintedanib versus placebo (HR, $0.38 ; \mathrm{P}=0.005)(16)$. On the basis of these results, PFD and nintedanib are conditional recommendation drugs for IPF patients on international 2015 IPF guideline (17). Raghu et al. conducted a post hoc subgroup analysis of patients with honeycombing on HRCT and/or confirmation of usual interstitial pneumonia (UIP) by biopsy versus patients without either, using pooled data from the INPULSIS trials. Among 1,061 IPF patients, $68.1 \%$ patients had honeycombing and/or biopsy, and $31.9 \%$ patients had no honeycombing or biopsy. In these subgroups, the adjusted annual FVC decline in patients treated with placebo were -225.7 and $-221.0 \mathrm{~mL} / \mathrm{year}$, and the nintedanib group were $117.0 \mathrm{~mL} /$ year (95\% CI, 76.3-157.8) and $98.9 \mathrm{~mL} /$ year (95\% CI, 36.4-161.5). Therefore, effect of nintedanib for IPF did not depend on whether honeycombing or not in these study patients (18). Advanced IPF patients have high incidence of pulmonary hypertension (PH). Pathogenesis of $\mathrm{PH}$ in IPF is hypoxic vasoconstriction, aberrant vessel remodeling, and destruction of the intra-alveolar vessel (19). Endothelin-1 play important role for endothelial injury, pulmonary vessel hypertrophy which can impact on PH. Therefore, many interstitial lung disease expert highlight on endothelin-1 receptor antagonist (ERA) for IPF-PH. BUILD-1 study was conducted for 158 IPF patients randomly received bosentan $(n=74)$ or placebo $(n=84)$. Bosentan demonstrated no significant benefit over placebo in the primary endpoint such as six-minute-walk distance (6MWD) up to 12 months. On the other hand, bosentan showed superiority for time to death or disease progression which was the secondary endpoint. (HR, 0.613; 95\% CI, $0.328-1.144 ; \mathrm{P}=0.119)$, which was more significant in a patient subgroup diagnosed by surgical lung biopsy (SLB) (HR, 0.315; 95\% CI, 0.126-0.789; $\mathrm{P}=0.009$ ). Bosentan showed benefit about trend of dyspnea and QoL until 12 months (20). This study did not satisfy primary endpoint and latest guideline show importance of chest highresolution computed tomography (HRCT) for diagnosis IPF. In addition, SLB have gradually decreased because of the spread of international guideline, aging and comorbidity of real-world patients. Therefore, indication of bosentan for biopsy-proven IPF patients do not match daily clinical practice. Another study of ERA with macitentan for IPF was conducted. Inclusion subjects were adults IPF within 3 years disease duration and a histological pattern of UIP on SLB. The median FVC change over 12 months was $-0.20 \mathrm{~L}$ in the macitentan arm and $-0.20 \mathrm{~L}$ in the placebo arm. Overall, no differences between treatments were demonstrated in physiologically and survival. Median drug exposures to macitentan and placebo were 14.5 and 15.0 months, respectively (21). Macitentan showed no significant difference of serial change of FVC which is the most important physiological surrogate maker for IPF. On the basis of these studies, strategy of ERA for IPF is abandoned. On the other hand, sildenafil is a phosphodiesterase-5 (PDE-5) inhibitor which have different mechanism from ERA and is approved for primary arterial hypertension. PDE-5 inhibitor is selective pulmonary vasodilator and may preferentially improve blood flow to well-ventilated regions of the lung of IPF patients. Therefore, sildenafil was attractive for the possibility of treatment of severe IPF. In the placebo-controlled Sildenafil Trial of Exercise Performance in Idiopathic Pulmonary Fibrosis (STEP-IPF) was conducted for advanced IPF patients with \%DLco less than 35\% (22). This study was a double-blind, randomized, placebo-controlled trial of sildenafil in two phases. The first period consisted of 12 weeks of a double-blind comparison between sildenafil and a placebo control. The primary outcome was the proportion of patients with an increase in the $6 \mathrm{MWD}$ of $20 \%$ or more. Main secondary outcomes were oxygenation changes, degree of dyspnea, and QoL. The second period was a 12week open-label evaluation consist of all patients receiving sildenafil. One hundred and eighty advanced IPF patients were recruited. The difference in the primary outcome was not significant, with 9 of 89 patients $(10 \%)$ in the sildenafil arm and 6 of $91(7 \%)$ in the placebo arm having an improvement of $20 \%$ or more in the $6 \mathrm{MWD}(\mathrm{P}=0.39)$. There were small but significant differences in arterial oxygenation, DLco, degree of dyspnea, and QoL favoring 
the sildenafil group. Primary outcome showed no signify difference. However, several parameters such as oxygenation, decline of \% DLco, clinical symptoms including dyspnea grade and QoL showed positive result. Therefore, next validation study was expected.

Kolb et al. published INSTAGE clinical trial in 2018 September issue in New England Fournal of Medicine (23). The purpose of this study was added effect of sildenafil with nintedanib for advanced stage of IPF patients. They randomly assigned, in a 1:1 ratio, IPF patients with DLco of $35 \%$ or less of the predicted value to receive nintedanib at a dose of $150 \mathrm{mg}$ twice daily plus sildenafil at a dose of $20 \mathrm{mg}$ three times daily (combination group) or nintedanib at a dose of $150 \mathrm{mg}$ twice daily plus placebo three times daily (monotherapy group) for 24 weeks. The primary end point was the trend of the total score on the SGRQ score at week 12 (the total score ranges from 0 to 100, with higher scores indicating worse health-related QoL). Secondary end points included measures of dyspnea and safety. A total of 274 IPF patients underwent randomization. The primary endpoint showed no significant difference [nintedanibplus-sildenafil group and the nintedanib group $(-1.28$ and -0.77 points, respectively; $\mathrm{P}=0.72$ ] at 12 weeks. In addition, both dyspnea and safety did not show any positive effect of sildenafil for these advanced IPF patients. This result has two sides interpretation. One aspect is sildenafil demonstrated no added effect rather than nintedanib alone for severe IPF patients. Other point of view is sildenafil had no harmful effect for advanced IPF patients who received nintedanib. There are several limitations of this study. First, study duration is 24 weeks, which is not enough period. Monitoring of dyspnea trend and physiological parameters change requires minimum 24 weeks. Therefore, more long period study will be warranted in the near future. Second, as author described, patient-reported outcome is subjective evaluation which may contribute to patient bias. SGRQ and The University of California, San Diego, Shortness of Breath Questionnaire (UCSD-SOBQ) are world-wide accepted questionnaire. However, there are still not objective compared to laboratory data or physiological parameters such as FVC and DLco. Third, SGRQ is a 50item questionnaire that assesses health-related QoL in patients with respiratory disease. UCSD-SOBQ is a 24item questionnaire assessing the severity and limitations of dyspnea during everyday activities. However, these items are quite many and complicated, which is associated timeconsuming. Therefore, introduction of these questionnaire for every hospital is not practical. Fourth, in terms of emphysema, the presence of emphysema was determined by the investigators on the basis of qualitative assessment of chest HRCT scans. But, they did not mention about threshold of emphysema extent of this study. Existence of emphysema is depending on subjective judgement of individual investigator. Interaction of emphysema with IPF is complicated and quite important issue for disease progressions. Majority of IPF patients are elderly smokers and often have emphysema. Combined pulmonary fibrosis and emphysema is proposed as one of the syndromes of IPF category (24). Emphysema is destroyed alveolar wall, which lead to destruction of alveolar wall vessels. IPF patients often show vascular remodeling and fibrotic destruction of vessels. Both IPF and emphysema show hypoxic vasoconstriction as disease progress. Based on these pathogeneses, both emphysema and IPF tend to show decline of DLco. Therefore, setting standardized threshold of emphysema extent is crucial for IPF clinical study. Generally, over $10 \%$ of emphysema extent is significant, setting less than $10 \%$ of emphysema out of total lung field is valid for next IPF study. Fifth, patients are diagnosed IPF based on 2011 international guideline. And the proportion of IPF patients who underwent SLB was not clear in this study. 2011 guideline divided into three groups of HRCT UIP pattern such as definite, possible, and inconsistent. On the contrary, latest 2018 international IPF guideline proposed four category of HRCT UIP pattern such as definite, probable, indeterminate and alternative diagnosis like Fleischner Society White Paper (25). Therefore, target population of advanced IPF patients may be different depend on guideline. Without SLB, inclusion of both definite and probable UIP pattern patients will be reasonable. Sixth, in terms of evaluation of right ventricular dysfunction, they estimated the presence of any echocardiographic sign indicative of right heart dysfunction such as right ventricular systolic dysfunction, right ventricular hypertrophy, right ventricular dilatation, paradoxical septum motion, or right atrium enlargement. Among these findings, which is most significant determinant? how many findings are truly associated with right ventricular dysfunction? These questions remain unknown. In addition, echo findings are usually influenced on technician's art. So, reproducibility and validity are less robust. Seventh, they showed change of serum brain natriuretic peptide (BNP) over 24 weeks. BNP is falsely elevated with reduced renal function. Therefore, adjustment of renal function is required. Finally, FVC difference of $32.5 \mathrm{~mL}$ at 12 weeks and that of difference of $46.3 \mathrm{~mL}$ at 
24 weeks is questionable threshold for patient's dyspnea or health-related QoL. However, treatment with nintedanib plus sildenafil was associated with a lower risk of an absolute decline at least 5 percentage points of the predicted value of the FVC (\%FVC) or death than treatment with nintedanib alone $(31.4 \%$ vs. $50.7 \%$ of patients; HR, 0.56 ; $95 \%$ CI, 0.38 to 0.82 ) and a quarter of the patients in the combination group had a relative decline of at least $10 \%$ of the $\% \mathrm{FVC}$ or died, as compared with $36.8 \%$ of those in the nintedanib monotherapy group (HR, 0.68). These parameters were not target endpoint. However, their evidence may be meaningful for slowing the progression of IPF.

Sildenafil is not harmful for advanced IPF patients with less than $35 \%$ of \%DLco. Compared to ERA, sildenafil shows slightly promising result. In addition, sildenafil is not expensive rather than ERA and anti-fibrotic agent. Costeffective management strategy is acceptable. Latest 2018 international guideline stress on the importance of chest HRCT findings. Therefore, I hope more accurate selection of subgroup of IPF patients based on new guideline especially excluding the effect of emphysema will be important in the next clinical study.

\section{Acknowledgements}

None.

\section{Footnote}

Conflicts of Interest: The author has no conflicts of interest to declare.

\section{References}

1. American Thoracic Society, European Respiratory Society. American Thoracic Society/European Respiratory Society international multidisciplinary consensus classification of the idiopathic interstitial pneumonias. Am J Respir Crit Care Med 2002;165:277-304.

2. Raghu G, Collard HR, Egan JJ, et al. ATS/ERS/JRS/ ALAT Committee on Idiopathic Pulmonary Fibrosis. An Official ATS/ERS/JRS/ALAT statement: Idiopathic pulmonary fibrosis: evidence-based guidelines for diagnosis and management. Am J Respir Crit Care Med 2011;183:788-824.

3. Wells AU. Managing diagnostic procedures in idiopathic pulmonary fibrosis. Eur Respir Rev 2013;22:158-62.

4. Schmidt SL, Tayob N, Han MK, et al. Predicting pulmonary fibrosis disease course from past trends in pulmonary function. Chest 2014;145:579-85.

5. du Bois RM, Weycker D, Albera C, et al. Forced Vital Capacity in Patients with Idiopathic Pulmonary Fibrosis. Am J Respir Crit Care Med 2011;184:1382-9.

6. Nishiyama $\mathrm{O}$, Taniguchi H, Kondoh $\mathrm{Y}$, et al. Healthrelated quality of life in patients with idiopathic pulmonary fibrosis. What is the main contributing factor? Respir Med 2005;99:408-14.

7. Kondoh Y, Taniguchi H, Yokoi T, et al. Cyclophosphamide and low-dose prednisolone in idiopathic pulmonary fibrosis and fibrosing nonspecific interstitial pneumonia. Eur Respir J 2005;25:528-33.

8. Miyazaki Y, Azuma A, Inase N, et al. Cyclosporine A combined with low-dose corticosteroid treatment in patients with idiopathic pulmonary fibrosis. Respir Investig 2015;53:288-95.

9. Lederer DJ, Martinez FJ. Idiopathic pulmonary fibrosis. N Engl J Med 2018;378:1811-23.

10. Taniguchi H, Ebina M, Kondoh Y, et al. Pirfenidone Clinical Study Group in Japan. Pirfenidone in idiopathic pulmonary fibrosis. Eur Respir J 2010;35:821-9.

11. Kim ES, Keating GM. Pirfenidone: a review of its use in idiopathic pulmonary fibrosis. Drugs 2015;75:219-30.

12. Richeldi L, Costabel U, Selman M, et al. Efficacy of a tyrosine kinase inhibitor in idiopathic pulmonary fibrosis. N Engl J Med 2011;365:1079-87.

13. Bando M, Yamauchi H, Ogura T, et al. Japan Pirfenidone Clinical Study Group. Clinical Experience of the Longterm Use of Pirfenidone for Idiopathic Pulmonary Fibrosis. Intern Med 2016;55:443-8.

14. Ogawa K, Miyamoto A, Hanada S, et al. The Efficacy and Safety of Long-term Pirfenidone Therapy in Patients with Idiopathic Pulmonary Fibrosis. Intern Med 2018;57:2813-8.

15. Wollin L, Wex E, Pautsch A, et al. Mode of action of nintedanib in the treatment of idiopathic pulmonary fibrosis. Eur Respir J 2015;45:1434-45.

16. Richeldi L, du Bois RM, Raghu G, et al. Efficacy and safety of nintedanib in idiopathic pulmonary fibrosis. $\mathrm{N}$ Engl J Med 2014;370:2071-82.

17. Raghu G, Rochwerg B, Zhang Y, et al. An Official ATS/ ERS/JRS/ALAT Clinical Practice Guideline: Treatment of Idiopathic Pulmonary Fibrosis. An Update of the 2011 Clinical Practice Guideline. Am J Respir Crit Care Med 2015;192:e3-19.

18. Raghu G, Wells AU, Nicholson AG, et al. Effect of Nintedanib in Subgroups of Idiopathic Pulmonary Fibrosis 
by Diagnostic Criteria. Am J Respir Crit Care Med 2017;195:78-85.

19. Patel NM, Lederer DJ, Borczuk AC, et al. Pulmonary hypertension in idiopathic pulmonary fibrosis. Chest 2007;132:998-1006.

20. King TE Jr, Behr J, Brown KK, et al. BUILD-1: a randomized placebo-controlled trial of bosentan in idiopathic pulmonary fibrosis. Am J Respir Crit Care Med 2008;177:75-81.

21. Raghu G, Million-Rousseau R, Morganti A, et al. Macitentan for the treatment of idiopathic pulmonary fibrosis: the randomised controlled MUSIC trial. Eur Respir J 2013;42:1622-32.

Cite this article as: Kishaba T. Triple kinase inhibitor with phosphodiesterase-5 inhibitor for idiopathic pulmonary fibrosis. J Thorac Dis 2018;10(11):5974-5978. doi: 10.21037/ jtd.2018.10.84
22. Zisman DA, Schwarz M, Anstrom KJ, et al. A controlled trial of sildenafil in advanced idiopathic pulmonary fibrosis. N Engl J Med 2010;363:620-8.

23. Kolb M, Raghu G, Wells AU, et al. Nintedanib plus Sildenafil in Patients with Idiopathic Pulmonary Fibrosis. N Engl J Med 2018. [Epub ahead of print].

24. Cottin V, Nunes H, Brillet PY, et al. Combined pulmonary fibrosis and emphysema: a distinct underrecognised entity. Eur Respir J 2005;26:586-93.

25. Raghu G, Remy-Jardin M, Myers JL, et al. Diagnosis of Idiopathic Pulmonary Fibrosis. An Official ATS/ERS/JRS/ ALAT Clinical Practice Guideline. Am J Respir Crit Care Med 2018;198:e44-68. 